\title{
Zweiter Teil, drittes Buch: Erlösung oder die ewige Zukunft des Reichs und Schwelle
}

\author{
Renate Schindler
}

Franz Rosenzweig (1886-1929) wurde in Kassel geboren. Er gehörte dem emanzipiert-liberalen deutschen Judentum an und war im Ersten Weltkrieg als Soldat in Mazedonien stationiert. Ab 1916 befand er sich an der Balkanfront und entwarf hier - teilweise auf Feldpostkarten und in den Schützengräben sein philosophisches Jahrhundertwerk, den Stern der Erlösung (veröffentlicht $1921)^{186}$. Geprägt von traumatischen Erfahrungen schreibt er gegen Ende des Krieges an seine Mutter und an seinen engen Freund und Cousin Rudolf Ehrenberg:

[...]ich könnte einen ruhigen Winter gut brauchen, denn ich habe ein Buch [...] angefangen, mein System, muss ich wohl sagen [...] es hat drei Teile zu je drei Büchern [...]. Es ist vor 14 Tagen plötzlich da gewesen und seitdem sitze ich unter einer Dusche von Gedanken. ${ }^{187}$

${ }^{186}$ Im Folgenden werden dem laufenden Text Stellen aus diesem Werk in Klammern eingefügt - Zitate gemäss Franz Rosenzweig, Der Stern der Erlösung, Frankfurt a.M. 1993.

187 GS II, Brief vom 4.9. 1918 an Rudolf Ehrenberg und seine Mutter Adele Rosenzweig; vgl. auch Martin Buber, Franz Rosenzweig, in: ders., Der Jude und sein Judentum. Gesammelte Aufsätze und Reden, Gerlingen 1993, 801:

How to cite this book chapter:

Schindler, R. 2021. Zweiter Teil, drittes Buch: Erlösung oder die ewige Zukunft des Reichs und Schwelle. In: Brasser, M., Bojanić, P. and Ciglia, F. P. (eds.) The Star for Beginners: Introductions to the Magnum Opus of Franz Rosenzweig. Pp. 113-130. London: Ubiquity Press. DOI: https://doi.org/10.5334/bco.j. License: CC-BY 
In der Endfassung einer der wichtigsten glaubensphilosophischen Abhandlungen des 20. Jahrhunderts befindet sich das Kapitel über die Erlösung oder die ewige Zukunft des Reichs als drittes Buch im zweiten Teil von Rosenzweigs Hauptwerk. Unter der Überschrift Schwelle markiert es zugleich den Übergang in den dritten Teil der Studie. Wie der Begriff der ,ewigen Zukunft auf das Thema der Erlösung verweist, so kündigen auch die Titel der drei anderen Teilbände das systematisch entfaltete Verhältnis zwischen Zeit und Ewigkeit an. Es zieht sich als roter Faden durch die gesamte Abhandlung hindurch. Denn die spekulative Kosmologie des Stern der Erlösung wendet sich vehement gegen die Tradition des kontinentalen Denkens „von Jonien bis Jena“ (13) oder „von Thales bis Hegel“" (15), in der die Zeiterfahrung des einzelnen sterblichen Menschen dem Herrschaftsanspruch eines allgegenwärtigen und zeitlosen Begriffsapparats ausgeliefert worden sei. ${ }^{188}$

Rosenzweigs Kritik am traditionellen Rationalitätsbegriff und dessen Auswirkungen auf die Krise der europäischen Kultur setzt dagegen einen Begriff von Philosophie voraus, der für den „Weltanschauungs-, ja Standpunktsphilosophen" charakteristisch ist (117). Auf den Spuren Schellings, Schopenhauers und Nietzsches verteidigt Rosenzweig das benannte einzelne Individuum gegen die zeitlose „Identität von Denken und Sein“189. Denn er zeigt, dass in der Geschichte Gottes, der Welt und des Menschen verschiedene Zeiten und Ewigkeiten ineinander spielen: Die Ewigkeit im ersten Teil, der von der immerwährenden Vorwelt des antiken Heidentums handelt, ist von der Dauer und der bleibenden Zeit geprägt. Der zweite Teil stellt die Ewigkeit des Allzeiterneuerten und Sich-Erneuernden dar. Hier ist es das komplexe Gefüge der Zeiten und Ewigkeiten der Schöpfung (oder des immerwährenden Grundes der Dinge), der Offenbarung (oder der allzeiterneuerten Geburt der Seele) und der Erlösung (oder der ewigen Zukunft des Reiches), das in den lebendigen Beziehungen zwischen Gott, Welt und Mensch gründet und den Beginn der Weltgeschichte markiert. Im letzten Band geht es schliesslich um die von der wahren und eigentlichen Ewigkeit Gottes geprägte Überwelt.

In jeder dieser Welten und ihrem zwischen Zeiten, Ewigkeiten und Geschichte ausgespannten Horizont diskutiert Rosenzweig die Tatsächlichkeiten ambivalenter Grunderfahrungen von Leben und Sterben, Tod und Liebe, Sprechen und Schweigen sowie Glauben und Wissen. Er reflektiert sie mit Bezug auf

Buber teilt uns im Jahre 1930 mit, dass er für die Darstellung der besonderen Art, in der die damalige Zeit „die Katastrophen der historischen Wirklichkeiten“ als „Krisen des menschlichen Verhältnisses zur Wirklichkeit“ erfahren hat, „kein grösseres und deutlicheres Beispiel als das Franz Rosenzweigs" gefunden habe.

${ }^{188}$ GS III, 139-161, insbesondere 149-152; vgl. auch Renate Schindler, Zeit, Geschichte, Ewigkeit in Franz Rosenzweigs Stern der Erlösung, Berlin 2007, 27-68.

189 GS III, 151. 
die existentielle Bedeutung der Zeitphänomene theoretischer Philosophie, praktischer Philosophie und Theologie, um unsere Aufmerksamkeit auf deren logische und sprachliche Verknüpfung untereinander zu lenken.

Die Bahn der Zeitdimensionen der Weltzeit im zweiten Teil des Stern der Erlösung, die in der ewigen und allzeiterneuerten Gegenwart des geschichtlichen Ereignisses der Offenbarung ihr Zentrum findet, strahlt nicht nur in die ,erste Offenbarung' Gottes in der Vergangenheit der Schöpfung aus, sondern auch in die an der Zukunft ausgerichtete Welt der Erlösung. Hier rückt der „Zeiger der Weltuhr“ „[...] wie bei der Gestaltwerdung Gottes von der Schöpfung zur Offenbarung [...] bei der Gestaltwerdung der Seele von der Offenbarung zur Erlösung " weiter (236). Deren entscheidendes Merkmal besteht darin, dass die Welt als menschliche Mitwelt entdeckt wird.

Um die Struktur des Kapitels über die Erlösung und deren Einbettung in das gesamte System von Rosenzweigs Hauptwerk erschliessen zu können, geht es im Folgenden zunächst um Rosenzweigs fundamentale Frage, die sich auf eine Interpretation des Verhältnisses von der in der Offenbarung geäusserten Liebe Gottes zu den Menschen und deren Liebe untereinander bezieht. (1)

Im Anschluss hieran werden die zentralen Thesen aufgegriffen, die Rosenzweig zur Tat der (Nächsten)Liebe sowie zu den Unterschieden und Gemeinsamkeiten zwischen dem Reich Gottes und der säkularisierten Welt aufstellt. Sie sind in den Zusammenhang einer Analyse der für die Erlösungsproblematik charakteristischen Sprache eingebettet und stellen auch deren kritisch gegen den Deutschen Idealismus gewandte Implikationen in einer Theorie der Kunst dar. (2)

Ein weiterer Schritt führt uns in das Zentrum des Textes, das in der Entwicklung des Begriffs messianischer Zukunft besteht (3). Sie leitet zu Rosenzweigs Ausführungen im kurzen Kapitel Schwelle über, das zugleich den Beginn des dritten Teils markiert und Grundzüge der eigen Überwelt Gottes beschreibt. (4)

\section{Die Schlüsselfrage: Wie hängt das Gebot der Nächstenliebe mit dem Gebot der Liebe zu Gott zusammen?}

\section{Die ersten Sätze des Buches über die Erlösung lauten}

Liebe Deinen Nächsten. Das ist, so versichern Jud und Christ, der Inbegriff aller Gebote. Mit diesem Gebot verlässt die mündig gesprochene Seele das Vaterhaus der göttlichen Liebe und wandert hinaus in die Welt. (229)

Rosenzweig knüpft hier an das bereits in den Ausführungen zum Offenbarungsgeschehen hervorgehobene erste Moment der Erlösungserfahrung an. Denn er nimmt den Gedanken der absoluten Gegenwart der wechselseitigen Liebe zwischen Gott und Mensch wieder auf und bestimmt sie als den Grund für die Liebe zum Nächsten: 
Indem die Liebe zum Menschen von Gott geboten wird, wird sie, weil Liebe nicht geboten werden kann ausser von dem Liebenden selber, unmittelbar auf die Liebe zu Gott zurückgeführt. Die Liebe zu Gott soll sich äussern in der Liebe zum Nächsten [...]. Die gottgeliebte Seele allein kann das Gebot der Nächstenliebe zur Erfüllung empfangen. Gott muss sich erst zum Menschen gekehrt haben, ehe der Mensch sich zu Gottes Willen bekehren kann. (239f.)

Der Leser wird gleich zu Beginn der Ausführungen zum Thema der Erlösung mit der Frage konfrontiert, wie der im Deuteronomium (6,5) angeführte Imperativ $^{190}$, das in der Offenbarungsgegenwart Gottes zu vernehmende „Liebe mich“, notwendigerweise zu einem weiteren Liebesgebot führt. Rosenzweig fasst hier die wichtigste These des Kapitels über die Offenbarung zusammen: Das „Urgebot“ der Liebe zu Gott das „in allen einzelnen Geboten mittönt und sie erst aus der Starrheit von Gesetzen zu lebendigen Geboten schafft“ (ebd.) sei „der Inbegriff, das worin alle [...] Gebote schliesslich münden“ und „gebietet [...] die einzige Liebe [...], die geboten werden kann“" (229). ${ }^{191}$

Emmanuel Lévinas hebt es als deutlich jüdischen Zug in Rosenzweigs Denken hervor, dass unter der Offenbarung Gottes nicht nur der Beginn des Werks der Erlösung verstanden wird. Denn sie ist zugleich ein Werk, dessen Vollendung davon abhängt, ob es dem Menschen gelingt, den ihm in der Nächstenliebe aufgegebenen sittlichen Auftrag zu verwirklichen:

Das Judentum, in dem die Erlösung untrennbar mit den Geboten zusammenhängt, bedeutet keineswegs die Last des Gesetzes, sondern genauer gesagt Liebe. Dass das Judentum durch die Gebote gekennzeichnet wird, bezeugt die Erneuerung der Liebe Gottes zu jedem Menschen in jedem Augenblick. Ohne sie hätte die Liebe, die in den Geboten aufgetragen wird, dem Menschen überhaupt nicht aufgetragen werden können. ${ }^{192}$

Rosenzweigs entscheidende Frage lautet nun, wie sich das Urgebot der Liebe zu Gott, „damit [...] vereint“ (229), dass die Liebe zum Nächsten ebenfalls

${ }^{190}$ Vgl. hierzu Robert Gibbs, The Grammar of the Laws, 3, 4 (zitiert nach dem Manuskript eines Vortrags auf dem Internationalen Rosenzweigkongress in Paris 2009). Gibbs widmet sich im Kontext der Bedeutung von Gesetz und Gebot im Judentum der Frage nach der Philologie in der Auszeichnung des Du im Gebot der Gottesliebe sowie dessen Übersetzungen in verschiedenen Ausgaben der Bibel.

191 Vgl. Schindler, a.O., 289ff.

${ }^{192}$ Emmanuel Lévinas, Entre deux mondes. Biographie spirutuelle de Franz Rosenzweig, in: La Conscience Juive - Données et Débats, Paris 1963, 121149. Hierzu kritisch Stéphane Mosès, La critique de la totalité dans la philosophie de Franz Rosenzweig, in: Les Études philosophiques 3 (1976), 351-366. 
geboten werden kann. Die folgende programmatische Formulierung bildet den Rahmen um die ganze Problematik der Erlösung:

Die Antwort auf dieses Bedenken könnte leicht in einem kurzen Wort vorweggenommen werden. Statt dessen sei ihr lieber das ganze Schlussbuch dieses Teils gewidmet. Denn sie enthält, so einfach sie ist, alles in sich, was die beiden vorangehenden Bücher noch offen lassen mussten. (ebd.)

Welche ,kurze Antwort' meint Rosenzweig? Und was wurde bisher ,offen' gelassen?

\section{Gliederung des Erlösungskapitels und zentrale Thesen}

\section{Die Tat der Liebe und die Liebe zum Nächsten}

Gemäss dem Verlauf von Rosenzweigs Argumenten im Blick auf sein gesamtes Buch wurde insbesondere das Thema der auf die Zukunft bezogenen Liebestat (229-243) noch nicht erschöpfend behandelt: Wenn sich der von Gott geliebte Mensch nun auf seinen Nächsten im Sinne des zu-nächst jeweils Nächsten als rea', plesios, proximus $(243,262)$ richtet, so geht es letztlich um die Strukturen verantwortlichen Handelns, die die Lebenspraxis einer säkularisierten sowie eine der Erlösung bedürftigen Welt erfordern.

Die aus der Nächstenliebe resultierende Tat kommt an verschiedenen zentralen Stellen zur Sprache:

a) Rosenzweig erörtert sie im Kontext der Bestimmung des Freiheitsbegriffs, den er als Grundlage des Gebots der Liebe zum anderen auffasst und von Kants Konzeption des in Autonomie begründeten Moralgesetzes absetzt (287f.). Er knüpft hier an die gleich in der Einleitung zum Stern der Erlösung aufgestellte These an, dass „[d]as Gesetz [...] dem Menschen, nicht der Mensch dem Gesetz gegeben [...] ist. (15). Diese neue Grundwahrheit besagt, dass die Vernunft und ihre Selbstgesetzgebung nicht mehr - wie bei Kant - das wichtigste Fundament der Moral bilden, sondern das in der Freiheit des menschlichen Willens und im Begriff des Nächsten verankerte ,Bedürfen des Anderen. Der Andere ist für Rosenzweig ein ,Du', insofern von ihm gesagt werden kann: „[E]r ist wie Du“ (267). Einen kritischen Blick wirft er auch auf die Auslegung des Gebots in der neben Juden- und Christentum dritten monotheistischen Weltreligion, im Islam (240-243). Die Erfüllung von Gottes Gebot durch die „Welttat“ erschöpfe sich bei den Anhängern dieser Glaubensgemeinschaft letztlich im reinen Gehorsam, dem Weg Allahs auch durch Glaubenskriege zu folgen. Die Vorschriften des islamischen Kriegs- und Eroberungsrechts weichen nach Rosenzweig grundsätzlich vom kanonischen und talmudischen Recht ab, 
weil sie den „Unterschied von Liebesgebot und Gesetzesgehorsam“ nicht erkennen (242). ${ }^{193}$

b) Im Zusammenhang mit der Interpretation der Sprache der Liebestat spielt es eine wichtige Rolle, wie sich der je andere dem einzelnen Menschen in der Grammatik des Pathos (254f.) und im Lob-und Dankgesang der Gemeinde (262) erschliesst.

c) Das ,Ich`öffnet sich dem ,Du' des Nächsten, indem sich die zunächst verschlossene Seele einer anderen Seele bedingungslos zuwendet und sie als Repräsentanten ,der Welt' wahrnimmt (267f.)

\section{Welt und Reich}

Es sind nach Rosenzweig folglich „Zwei Seiten“, von denen aus „an das verschlossene Tor der

Zukunft gepocht wird“: Vonseiten der menschlichen Seele, deren Herz und Gemüt ihren Weg zum Wirken am Nächsten sucht, und vonseiten des Lebens der Welt (254).

Denn die Welttat der Liebe bezieht sich im Erlösungsgeschehen auch auf die Vorwegnahme des Reiches und damit weniger unmittelbar als im Gebot der Gottesliebe, aber indirekt ebenfalls auf Gott, der in der Schöpfung den immerwährenden Grund der Dinge gelegt hat (243-254). Das Wissen um die Vergangenheit der Schöpfung als der „Pforte, durch die die Philosophie ins Haus der Theologie eintritt“ (104), spielt bereits in Rosenzweigs Ausführungen zum Begriff des gegen den Rationalismus der Aufklärung verteidigten Wahrheitsgehalts des Offenbarungswunders eine entscheidende Rolle. ${ }^{194}$ Es ist die Schöpfung, die den Pfeiler bildet, der das gesamte System des zweiten Teils des Stern der Erlösung trägt. Auf der Bahn der allzeiterneuerten Welt in der Geschichte von Gott, Welt und Mensch werden die theologischen Kategorien Offenbarung und Erlösung ausdrücklich auf die Schöpfung zurückgeführt. Sie ist erst dann vollendet, wenn sich das Individuum der Welt und der menschlichen Mitwelt so

${ }^{193}$ Vgl. Martin Brasser, Rosenzweig und die Karikatur des Islam. Negative Konstruktionen im interreligiösen Dialog, Vortrag, in: ders. (Hg. u.a.), Rosenzweig Jahrbuch 2 / Rosenzweig Yearbook 2, Kritik am Islam / Criticism of Islam, Freiburg 2007, 128-151 (hier zitiert nach der Manuskriptvorlage). Brasser hat die Gründe für Rosenzweigs geostrategische (1-6) sowie interreligiöse Interpretationen des Islam methodisch dargestellt, die bewusst auf eine ,Parodie ' und Karikatur dieser Religionsgemeinschaft zielen (6-13); Vgl. zur Ausrichtung von Rosenzweigs Darstellung des Islam auf den Offenbarungsbegriff hin im Stern der Erlösung auch Gesine Palmer, Der verkannte Islam, in: Wolfdietrich Schmied-Kowarzik (Hg.), Franz Rosenzweigs „Neues Denken“, Internationaler Kongress Kassel 2004, Band 2, Freiburg/München 2006, 1109-1118.

$194 \mathrm{Vgl}$. Schindler, a.O., $211 \mathrm{ff}$. 
zuwendet, dass er sie durch Taten der Liebe verändert, die sich an der Vorwegnahme des Reiches Gottes orientieren. Es gelte - so hiess es an früherer Stelle:

die Offenbarung selbst und ihre Einbindung und Begründung in die Zuversicht auf das Kommen des sittlichen Reichs der endlichen Erlösung, diesen ganzen heut als den eigentlichen Kern des Glaubens empfundenen Zusammenhang, den die Hoffnung zwischen den Begriffen Offenbarung und Erlösung stiftet, selber wieder einzubauen in den Begriff der Schöpfung. Auch Offenbarung, auch Erlösung sind eben in gewisser, noch nicht auseinanderzusetzender Weise Schöpfung. (114)

Hierauf bezieht sich Rosenzweig, wenn er nun die „Sonderstellung der Welt“ (244) hervorhebt, indem er sie als, unfertig' bezeichnet und als stets im Werden begriffenes lebendiges Reich Gottes, dessen Ewigkeit für alle Zukunft in der Gegenwart des Augenblicks vorweggenommen werden kann $(244,245,248-251) .{ }^{195}$

Weiterhin geht er in seinen methodischen Erläuterungen (255-257) davon aus, dass in wahrem Sinne „nur Einer“ der Erlöser sein kann (255), d.h. dass Gott „in viel stärkerem Sinn [...] der Erlöser [...] als er Schöpfer und Offenbarer ist". Denn Gott erlöst die Welt durch den Menschen, und diesen in der Welt. Er ist ein Dritter, der sich in der Erlösung von Mensch und Welt auch selbst erlöst (229-265).

Diese Welt der Erlösung ist nicht nur deutlich vom falschen Zauber abzugrenzen, der den plastischen Kosmos der Antike sowie seine Künste der Magie und Astrologie in der immerwährenden Vorwelt prägte (245-248). Die erlöste Welt unterscheidet sich auch grundsätzlich von der Entzauberung der Welt in der modernen Wissenschaft $(246,247)$ sowie vom Fortschrittsbegriff des Islam. Im Unterschied zum Judentum und Christentum verfügt er nach Rosenzweig über keine einheitliche Darstellung der Geschichte (251-253). Insbesondere sei „hier der Gedanke der Zukunft in der Wurzel vergiftet“, da er auf einem ins Unendliche reichenden Fortschrittsbegriff beruht. Im Islam werde verkannt, dass die wahre Zeit der Ewigkeit eine von Gott verliehene Gabe ist, die den Menschen dazu befähigt, das Ende in der Gegenwart vorwegzunehmen. Der Gedanke, dass das Reich Gottes „heute“ kommt, diese „Verewigung des Augenblicks“ erlischt laut Rosenzweig „in dem islamischen wie im modernen Begriff der Zeitalter“ (253). ${ }^{196}$ Denn wir können darauf vertrauen, dass das Walten und Wachsen des Gottesreichs für menschliche Augen sichtbar ist und in uns „trotz aller Enttäuschungen“ auch durch die Vermittlung der rabbinischen Lehre immer neue Hoffnung auf einen besseren Zustand der Welt erweckt (252).

${ }_{195} \mathrm{Vgl}$. Abschnitt 3 im vorliegenden Text.

196 Dies scheint insbesondere darauf zurückzuführen zu sein, dass Rosenzweig den Islam als „Anti-Offenbarungsreligion“ funktionalisiert - vgl. hierzu Brasser, a.O., 12. 


\section{Die Sprache der Erlösung}

In menschlichen Handlungen, die sich auf den Nächsten richten, entdeckt Rosenzweig eine sprachliche und grammatikalische Struktur, in der „Mensch und Welt $[\ldots]$ in unauflösbarer Wechselwirkung aufeinander und miteinander [...] wirken“ - das „Wirken entbindet die Tat aus dem Menschen, aber bindet die entbundene auch wieder hinein in die Welt". Gut könne die Welt nur „durch die Guten“ werden, da Welt und Mensch „nicht voneinander zu lösen“ sind und daher nur miteinander erlöst werden können $(254,255)$.

Wie Rosenzweig in seinem Aufsatz über Das neue Denken (1925) schreibt, der im Untertitel Einige nachträgliche Bemerkungen zum, Stern der Erlösung ankündigt, herrscht in der Erlösung „die Sprache des Chors “197. In Analogie hierzu schreibt er in seinem Hauptwerk:

[D]ie Erlösung der Seele an den Dingen, der Dinge durch die Seele geschieht im gleichatmenden Zwiegesang der beiden, im Satz, der aus den Stimmen der beiden Worte zusammenklingt. (255)

In der sprachlichen Struktur der Lob- und Dankgesänge, der Psalmen und im liturgischen Leben der jüdischen Gemeinde zeigt sich die Erwartungshaltung gegenüber dem zukünftigen Kommen des Reichs der Erlösung (255-281).

Den Ausführungen Rosenzweigs zum Chor der Erlösung geht im ersten Teil seines Hauptwerks eine Theorie über den Chor der (antiken) Tragödie vorher, im dritten Teil greift er die Bedeutung des Chores in der Kirchenmusik auf (401ff.). Man kann geradezu davon sprechen, dass sich Rosenzweigs Ästhetik von der „Warte" des Chores wie von einer Festung aus erschliessen lässt. ${ }^{198}$ Das Wort „Chor" selbst kommt im Stern der Erlösung zwar nur elfmal vor. ${ }^{199}$ Aber es steht jeweils in allen drei Teilen seines Systems der Philosophie im Kontext von Interpretationen der Kunst und des Schönen, in denen Rosenzweig seine Gegnerschaft zur idealistischen Ästhetik betont.

Auch in der Bestimmung der Bedeutung des gemeinsamen Gesangs der jüdischen Gemeinde und deren grammatikalischen Charakteristika bezieht sich Rosenzweig auf das wichtigste Merkmal seines neuen Denkens als eines Sprachdenkens. Die Sprache als Organon existentiell erfahrbarer Zeitlichkeit und sinnliches Medium der im Sprechen, Miteinander-sprechen und Hören zu erkennenden Wirklichkeit untergräbt den in der idealistischen Tradition der

197 Rosenzweig, a.O., 151.

198 Luca Bertolino, Die Rolle des Chors in Franz Rosenzweigs Stern der Erlösung, in: Martin Brasser, Hans Martin Dober (Hg.), Rosenzweig Jahrbuch 5 / Rosenzweig Yearbook 5, Wir und die Anderen / We and the Others, Beiträge zum Kongress der Internationalen Rosenzweig-Gesellschaft in Paris vom 17.-20.Mai 2009, Teil I, Freiburg 2010, 141-159, hier 141.

199 Ebd. 
Philosophie unbedacht vorausgesetzten Anspruch eines Apriori der Vernunft und ihrer sich selbst genügenden begrifflichen Logik.

Im Rekurs auf die beiden vorangegangenen Bücher des zweiten Teils erinnert Rosenzweig an die Sprachform der Erzählung, in der er auf den Spuren Schellings die „Sätze der Geschichte“200 der Vergangenheit der Schöpfung in der dritten Person Singular dargestellt hat: „Er schuf, er sprach, er schied und so fort. Vergangenheit und ,er." So fasst Rosenzweig seine Analyse des Buches Genesis zusammen (168). ${ }^{201}$ Die Offenbarung dagegen ist von der Präsenz des Dialogs in der Liebeserfahrung eines Ichs und eines Du geprägt und wird am Beispiel des Hohelieds veranschaulicht; Gott eröffnet das Wechselgespräch mit dem Menschen sowie dasjenige zwischen Mann und Frau, „das zwischen zweien hin und her geht“. Im Ereignis der Erlösung jedoch „tritt die Grammatik [...] als strophisch sich steigernder Gesang [...]" in den Vordergrund und wird als Augenblick messianischer Zukunft erlebt (258). Es ist die Gemeinschaft der ,Wir ‘ als der Mitglieder der jüdischen Gemeinde, die an der Liturgie teilnehmen und gemeinsam ihre Stimme erheben (278-281). Der messianische Gehalt der Sprache der Erlösung kommt auch im Gebet zum Ausdruck, das „die Erfüllung all dessen ist, worum gemeinsam gebetet werden kann“ und die Zukunft der Verheissung in die Gegenwart vorwegnimmt. ${ }^{202}$

Die beiden Nominative ,Mensch' und ,Welt' sowie die zu ihnen gehörenden Akkusative bilden das mächtige Unisono der „Wir alle“; Gott, dem der Gesang gewidmet ist, steht im Dativ (259f.). Die einzelnen singulären Stimmen des ,Ich' und ,Du' ermutigen sich gegenseitig und gelangen vom Sprachmodus des Dual in denjenigen der von der Pluralität zu unterscheidenden Allheit:

Im Wir also hebt die Schlussstrophe des Gesangs der Erlösung an; im Kohortativ hatte er mit dem Aufruf der Einzelnen, die aus dem Chor

${ }^{200}$ Vgl. hierzu Wolfdietrich Schmied-Kowarzik, Vom Totalexperiment des Glaubens. Kritisches zur positiven Philosophie Schellings und Rosenzweigs, in: ders. (Hg.), der Philosoph F. Rosenzweig (1886-1929), Internationaler Kongress Kassel 1986, Band 2, Freiburg/München 1988, 771-798; Rosenzweig konzipiert den Systembegriff im Stern der Erlösung, dem die Frage der Vereinbarkeit von ,System und Offenbarung' zugrundeliegt, in steter Auseinandersetzung mit Schellings Begriff von ,positiver' und ,negativer Philosophie; den Begriff der Erzählung entnimmt er Schellings Philosophie über die Weltalter, über die allgemeine Geschichte göttlichen Lebens - vgl. hierzu Schindler, a.O.,62-68, 229-246.

201 Vgl. zur Analyse der Sprache Rosenzweigs im Horizont des hermeneutischen Prinzips im Umgang mit Bibel und Talmud Gérard Bensussan, Parole, langage et temporalités, in: J.E. Marquet (Hg.), Revue de Métaphysique et de Morale 4 (2000), 461-478.

202 Donatella di Cesare, Die Messianität der Sprache, in: Wolfdietrich SchmiedKowarzik, a.O., 862-871, hier 867. 
hervortraten, und den Responsen des Chors darauf begonnen; im Dual ging es in einem zweistimmigen Fugato, an dem sich immer neue Instrumente beteiligten, fort; im Wir endlich sammelt sich alles zum choralmässig gleichen Takt des vielstimmigen Schlussgesangs. (264) ${ }^{203}$

Das Sprachdenken impliziert, wie Rosenzweig in seinen nachträglichen Bemerkungen schreibt, all diese Modalitäten der Sprache, weil es „überhaupt vom Leben des anderen“ lebt, „mag der nun Hörer der Erzählung sein oder der Antwortende des Zwiegesprächs oder der Mitsprecher des Chors”. ${ }^{204}$

Die „Chorform" der Erlösung vereint Mensch und Welt im Lobgesang auf Gott, der die Welt erschaffen hat und im Dank dafür, dass er seine Liebe offenbart hat. Der „Urgesang“ der Erlösung gipfelt im „Stammsatz“: „[E]r ist gut“, der als ein „denn er ist gut““ (258) zu verstehen ist (Ps 106,I, 118,I,29, 136,I) und die Stammworte der Schöpfung und Erlösung zusammenschliesst. Der Schöpfung wird ausschliesslich das Prädikat „gut“ verliehen. ${ }^{205}$ Am Beginn der Offenbarung steht das Ich Gottes, welches im Imperativ des Liebesgebots das Du des Menschen erweckt, indem er ihn bei seinem Namen anruft. ${ }^{206}$ Nun jedoch geht es in der Gemeinschaft der Zweistimmigkeit von Welt und Mensch um den Namen Gottes. ,Er ist gut' - dieser Satz der Erlösung bildet nach Rosenzweig

das Dach über dem Hause der Sprache, der an sich wahre Satz, der Satz, der wahr bleibt, einerlei wie er gemeint ist und aus welchem Munde er kommt. (258)

Anschaulich erläutert er im Anschluss an die These von der herausragenden Bedeutung dieses Satzes:

Dass zwei mal zwei vier ist, kann unwahr werden, etwa wenn man es einem Papagei gelehrt hat und der es nun „spricht“; denn was ist dem Papageien die Mathematik? Aber der Satz, dass Gott gut ist, kann selbst in diesem skurrilsten aller möglichen Fälle seines Lautwerdens keine Unwahrheit werden; denn auch den Papageien hat Gott geschaffen, und auch auf ihn geht schliesslich seine Liebe. (Ebd.)

203 Bertolino, a.O., 151.

204 GS III, 151.

205 Wolfdietrich Schmied-Kowarzik hat herausgearbeitet, dass Rosenzweig die in Schellings Schöpfungsthematik der Weltalter angesprochenen negativen Aspekte kreatürlichen Daseins und deren politisch-gesellschaftliche Auswirkungen ausser acht lässt. Rosenzweig, der einerseits fundamentalethisch die Verantwortlichkeit des Einzelnen gegenüber der Geschichte stark macht, spricht mit Bezug auf die Schöpfung nicht vom Leid und den Verfehlungen der Menschen gegenüber sich selbst, gegenüber der Natur und mit Bezug auf den Glauben - vgl. ders., a.O., 795ff., 789.

${ }^{206}$ Vgl. hierzu Schindler, a.O., 288. 


\section{Die Theorie der Kunst}

In der Vergötterung der Kunst und im Verwerfen des „geschaffenen Gottesgartens der Sprache" (162f.) zeigt sich nach Rosenzweig die Armut des idealistischen Denkens und seiner Logik, die daher ebenso wie die Ästhetik einer vollkommenen Erneuerung bedarf. ${ }^{207}$

Der wahre Reichtum lebendiger alltäglicher Erfahrungen und Erkenntnisse wurde im Hortus conclusus der Kunstauffassung des Idealismus ignoriert. Wenn Kultur an die Stelle der Natur trete und das Kunstwerk nur als „bewusstloses Erzeugnis des Geistes gilt“ ${ }^{208}$ so ist der Mensch dazu verurteilt, gegenüber der werdenden Welt der Offenbarung blind und taub zu sein.

Die Lehre von der Kunst, ${ }^{209}$ die im zweiten Teil des Stern der Erlösung lediglich als notwendige „Episode“ $(213,221)$ bezeichnet wird, nimmt jedoch in Rosenzweigs Werk einen beachtlichen Raum ein. Sie geht weit über die Darstellung der „klassischen“ Kunst hinaus, da Rosenzweig ihre tiefreichende Verbindung zum lebendigen Dasein aufzeigt und ihr zutraut, den Menschen in den verlorenen Gottesgarten zurückzuführen. Diese These findet in der Soziologie der Kunst im dritten Teil des Stern der Erlösung ihre Bestätigung (393-397, 399-403, 412-415) und zeigt sich in der Bedeutung, die Rosenzweig der Kunst in der Liturgie zuspricht: Ihre gesellschaftliche Rolle besteht darin, das Bedürfnis nach Religiosität widerzuspiegeln, und sie stellt - so heisst es auf der "Schwelle“ zum dritten Teil - die Nähe des Menschen zur „ewige[n] Überwelt" Gottes her (293). ${ }^{210}$ Insofern kommt der Kunst im neuen Denken die entscheidende Aufgabe zu, als angewandte Kunst insbesondere der Liturgie die klassische und idealistische Auffassung vom reinen und „zweckfreien Wohlgefallen "211 am Schönen zu bereichern und zu überwinden.

Im Kapitel über die Erlösung umfasst die Theorie der Kunst (270-277) deren wesentliche Elemente und Bereiche: Rosenzweig untersucht den Urheber als Künstler, Mensch, Genie oder Dichter (272f. vgl. 165f, 215), das Werk in seinen epischen, lyrischen oder dramatischen Ausdrucksformen (272f., vgl. 216f.) und das Publikum bzw. den Betrachter (270-272) in ihrer jeweiligen Rolle, die sie in der Systematik von Bildkunst, Tonkunst und Dichtung spielen (273-276; vgl. $217-221) .{ }^{212}$

${ }^{207}$ GS III, 140.

${ }^{208}$ Bertolino, a.O.,144.

${ }^{209}$ Ebd., 142.

${ }^{210}$ Ebd., 144, 145.

${ }^{211}$ Ebd., 142: Bertolino verweist darauf, dass sich Rosenzweig mit dem Begriff vom „Zweckfreien Wohlgefallen“ vermutlich auf Immanuel Kant bezieht - vgl. ders., Kritik der Urteilskraft (1790), Akademie-Ausgabe, Bd.5, 204f., 209f., 354.

${ }^{212}$ Umfassend wird Rosenzweigs Ästhetik u.a. in folgenden Beiträgen dargestellt: Stéphane Mosès, L’esthétique de Franz Rosenzweig, in: O. Mongin (Hg. u.a.), Franz Rosenzweig, Les Cahiers de la nuit surveillé 1 (1982), 119-135; Jules Simon, Rosenzweig's Messianic Aesthetics, in: Schmied-Kowarzik, 


\section{Das Zentrum des Textes: Messianische Erfahrung zukünftiger Ewigkeit}

Wenn man sich Rosenzweigs Schlüsselfrage nach dem Zusammenhang zwischen dem Gebot der Gottesliebe und demjenigen der Liebe zum Nächsten noch einmal vor Augen hält, so besteht der Kern seiner Ausführungen in den Facetten der Entwicklung des im Horizont zukünftiger Ewigkeit bestimmten Erlösungsbegriffs: Das Wirken des Einzelnen als Nächstenliebe in einer stets im ,Werden' zu verstehenden Welt in ihrer säkularisierten Form sowie in der jüdischen Liturgie, die sprachlichen Grundlagen der ,Wechselwirkung' zwischen Mensch und Welt im allgemeinen sowie im Chorgesang - jeder dieser Themenbereiche bezieht sich auf die Vorwegnahme der Ankunft des Gottesreichs in der durch die Anwesenheit zukünftiger Ewigkeit erfüllten Gegenwart.

Wie geht Rosenzweig nun genauer betrachtet vor, um das Oszillieren zwischen Zeit und Ewigkeit, welches dem Aufbau seines ganzen Werkes das systematische Gerüst liefert, im Kontext des Erlösungsgeschehens verständlich zu machen?

\section{Das Heute, der Augenblick und das ausserzeitliche ,Wachstum' des Gottesreiches}

Bereits in einer Passage des Kapitels über die Offenbarung setzt Rosenzweig die Welt und Mensch erlösende Liebe Gottes mit der Ewigkeit im ,Heute' gleich und leitet daraus drei Thesen ab, die verschiedene Aspekte des Begriffs einer messianischen Zukunft zueinander in Beziehung setzen:

[...] Gottes Liebe [...] ist immer im Heute und ganz im Heute, aber alles tote Gestern und Morgen wird in dieses sieghafte Heute einmal verschlungen, diese Liebe ist der ewige Sieg über den Tod; die Schöpfung, die der Tod krönt und schliesst, kann ihr nicht Stand halten; sie muss sich ihr ergeben in jedem Augenblick und darum schliesslich auch in der Fülle aller Augenblicke, in der Ewigkeit. (182)

Die als Ewigkeit zu verstehende Gegenwart wird hier positiv bewertet und von den zeitlichen Dimensionen der Vergangenheit und derjenigen leeren Zukunft abgegrenzt, welche nicht die des ankommenden Messias sind. Der „ewige Sieg über den Tod" bedeutet zweitens, dass der Erlösung insofern Vorrang vor der Schöpfung zukommt, als der in dieser , mitgeschaffene' Tod definitiv überwunden werden kann. Und drittens spricht Rosenzweig von der Ewigkeit als der

\footnotetext{
a.O., 407-418; Annemarie Meyer, Die Bedeutung der Kunst in Franz Rosenzweigs Werk, in: Werner Licharz (Hg. u.a.), Franz Rosenzweig und Hans Ehrenberg. Bericht einer Beziehung, Frankfurt a.M. 1986, 35-54.
} 
„Fülle aller Augenblicke“, also von einer im umfassenden Sinn erfüllten Lebenszeit, die auf eine andere Zukunftsdimension als die des ,toten Morgen' anspielt.

In der im Titel der Erlösungstheorie angesprochenen ewigen Zukunft des Reichs überschneiden sich diese drei Aspekte und werden im Verlauf der Argumentation durch folgende Gedankengänge ergänzt: Rosenzweig holt zunächst die ewige Zukunft in die Gegenwart ein und bezeichnet sie als Dauer, in der alle lebendige Kreatur „dem Tode [...] widersteht [...]“. Als ,kreatürlich` werden nicht „bloss Lebewesen, sondern auch Institutionen, Gemeinschaften, Gefühle, Dinge, Werke - alles" bestimmt. (248). Hier scheint der Autor auf die natürliche oder physikalische Erfahrung gegenwärtiger Zeit anzuspielen. Sie besteht jeden Augenblick auf der ständigen Spannung zwischen Leben und Tod, die einem immer gleichen Wechsel von Veränderungen unterliegt. Der Focus liegt jedoch auf der von der ewigen Zukunft durchdrungenen Gegenwart, welche dem tödlichen sukzessiven Zeitfluss des Lebens abgerungen wird. Nach Gershom Scholem begegnen wir hier dem Moment der immer, d.h. der ewig gegenwärtigen Zukunft der Erlösung, die auch die Kabbalisten als „,die immer kommende Welt", als den „olam haba“, gedeutet haben. ${ }^{213}$ Es ist dieser Zukunftsbegriff, der die Bedeutung der messianischen Erfahrung zeitlicher Ewigkeit am deutlichsten zum Ausdruck bringt. Denn er verweist unmittelbar auf die geschichtsphilosophische Idee, dass die diesseitige Welt mit dem Anbruch des Reiches „ganz lebendig werden“ kann, da „das Lebendige [...] nach Ewigkeit [...] verlangt [...]“ (249). Die für Rosenzweigs Zukunftsbegriff aufschlussreichste Passage schliesst sich direkt an die These von der Ewigkeit alles Lebendigen an:

[...] das Reich, die Verlebendigung des Daseins, kommt von Anfang an, es ist immer im Kommen. So ist sein Wachstum notwendig. Es ist immer zukünftig, aber zukünftig ist es immer. Es ist ebenso schon da wie zukünftig. Es ist einfürallemal noch nicht da. Es kommt ewig. Ewigkeit ist nicht eine sehr lange Zeit, sondern ein Morgen, das ebensogut heute sein könnte. Ewigkeit ist eine Zukunft, die, ohne aufzuhören Zukunft zu sein, dennoch gegenwärtig ist. Ewigkeit ist ein Heute, das aber sich bewusst ist, mehr als Heute zu sein. Und wenn also das Reich ewig kommt, so bedeutet das, dass zwar sein Wachstum notwendig ist, aber dass das Zeitmass dieses Wachstums nicht bestimmt ist, ja genauer: dass das Wachstum gar kein Verhältnis zur Zeit hat. Ein Dasein, das einmal ins Reich eingegangen ist, kann nicht wieder herausfallen, es ist unter das Einfürallemal getreten, es ist ewig geworden. (250)

Hervorzuheben ist demnach vor allem derjenige Zukunftsbegriff, der die Ewigkeit des Reiches als in der Gegenwart anwesende Zukunft so bestimmt, dass sie noch etwas qualitativ anderes ist als die Ewigkeit des Augenblicks, welche den

${ }^{213}$ Gershom Scholem, Franz Rosenzweig und sein Buch Der Stern der Erlösung, Nachwort, in: SE, 525-549, hier 544. 
natürlichen Zeitfluss sprengt. Denn das Wachstum des Reiches Gottes geschieht zwar notwendigerweise, hat jedoch kein ,Zeitmass'. Dies expliziert Rosenzweig in den drei Sätzen, welche die Zeitlichkeit der Ewigkeit bestimmen: Sie ist nicht die leere Unendlichkeit der, sehr langen' Zeit, sondern die Möglichkeit des Morgen im Heute. Trotz dieser potentiellen Gegenwärtigkeit bleibt die Ewigkeit zukünftig; sie durchsetzt zwar die Gegenwart, geht aber nicht in ihr auf. Und obwohl die Ewigkeit in der Zukunft ist, welche nie aufhört, ist sie in einem über die Unmittelbarkeit des Augenblicks hinausweisenden Heute erfassbar.

Es ist diese Form der Ewigkeit, die Rosenzweig meint, wenn er über „das geniale Kapitel“ in der Ethik des reinen Willens (1904) seines Lehrers Hermann Cohen schreibt, „das vom Ideal handelt“. Hier betont er, dass Cohens Messianismus „eben keine Ausflucht der Trägheit“ sei, „die das Ziel ins Unendliche rückt, um sich inzwischen im Endlichen in zielloser Behaglichkeit einzurichten“. Vielmehr sei „Ewigkeit für Cohen „grade nicht die Summe aller Zeit“, sondern die „auf den Augenblick bezogene, in seiner ,Nussschale‘ [...] vollzogene fernste Zukunft". ${ }^{214}$

\section{Gegenwart und das Warten auf das Ende der Zeit}

In der zweiten und dritten Bestimmung der Ewigkeit kommt ein für Rosenzweigs Erlösungstheorie grundlegendes Paradox zum Ausdruck, das im Spannungsfeld zwischen Theologie und Existenzphilosophie anzusiedeln ist: Aus der Sicht des immer kommenden, ausserzeitlichen Reiches Gottes wird die ewige Zukunft der Ewigkeit betont, vom Standpunkt des sterblichen Menschen dagegen deren Gegenwart. ${ }^{215}$ Zukünftig kann die Ewigkeit für die menschliche Erfahrung nur sein, wenn sie in der Gegenwart erwartet, und als Ende der Zeit in ihr vorweggenommen wird:

[...] zur Zukunft gehört vor allem das Vorwegnehmen, dies, dass das Ende jeden Augenblick erwartet werden muss. Erst dadurch wird sie zur Zeit der Ewigkeit [...]. Dass jeder Augenblick der letzte sein kann, macht ihn ewig. Und eben dass jeder Augenblick der letzte sein kann, macht ihn zum Ursprung der Zukunft als einer Reihe, von der jedes Glied durch das erste vorweggenommen wird. Dieser Gedanke der Zukunft nun, dies, dass das Reich „mitten unter euch“ist, dass es „heute“ kommt, diese Verewigung des Augenblicks erlischt im islamischen wie

${ }^{214}$ GS III, 196f. Rosenzweig bezieht sich hier auf folgenden Satz Cohens in der Ethik des reinen Willens. Werke, Bd.7 (hg. H. Holzhey), Hildesheim/New York 1981, 424: „In jeder Nussschale liegt die Unendlichkeit; sie liegt nicht in ihr; aber sie vollzieht sich in ihr“.

${ }^{215}$ Vgl. hierzu Eva Birkenstock, Heisst philosophieren sterben lernen? Antworten der Existenzphilosophie: Kierkegaard, Heidegger, Sartre, Rosenzweig, Freiburg/München 1997, $270 f$. 
im modernen Begriff der Zeitalter. Hier bilden die Zeiten zwar eine unendliche Reihe, aber unendlich ist nicht ewig, unendlich ist nur „immerzu“. (252f.)

Mit Bezug auf die Welt bedeutet dies, dass sie aus jeglichem starren Vergangenheitsbezug herausgelöst ist; durch die in die Gegenwart vorgezogene ewige Zukunft des Reiches Gottes betrachten wir sie als immer ,unfertig', da sie sich in einem ständigen Zustand des ,Werdens' befindet.

Stéphane Mosès stellt im Zuge der Ausarbeitung einer Phänomenologie des Wartens fest, dass Rosenzweigs Zukunftsbegriff insofern auf die religiöse Grunderfahrung zurückzuführen ist, dass die Menschen im Einbruch der mit Ewigkeit aufgeladenen Zukunft des Reiches Gottes „auf etwas radikal Neues“ warten. ${ }^{216}$ Dieses ragt aus dem Verlauf des natürlichen Zeitflusses heraus, es kann in dessen Binnenhorizont nicht erlebt werden. Denn die religiöse Zukunftserfahrung sei von der Vorstellung der berechtigten Hoffnung auf einen Zustand der Welt dominiert, der völlig anders als der jetzige ist und sofort eintreten kann. Während die Utopie das bezeichnet, was „was ganz am Schluss der Zeit" und am Ende eines Weges mit ungewissem Ziel liege, beziehe sich die spezifisch menschliche messianische Hoffnung auf eine erlöste Welt, die durch die Vorwegnahme der Ankunft des Endes „schon jetzt eintreten kann“. ${ }^{217}$

\section{,Messianische Ungeduld“}

Die menschliche Erfahrung der Zukunft wird für Rosenzweig also wesentlich durch die Erlösungssehnsucht bestimmt, die er in einem Brief an Gertrud Oppenheimer aus dem Jahre 1917 als messianische Ungeduld bezeichnet. Er erläutert anhand von zwei Beispielen, was darunter zu verstehen ist. Zuerst gibt er eine talmudische Legende wieder:

Ein Rabbi trifft am Eingang einer Höhle den Profeten Elias (bekanntlich Vorläufer des Messias, nach Maleachi, Schlussvers) und fragt ihn: Wo ist Messias? Drinnen in der Höhle. Da geht er hinein und findet Messias da sitzen. Da fragt er: wann kommst du, Herr? Messias antwortet: Heute. Da geht er fröhlich heraus und wartet bis zum Abend. Als aber Messias immer noch nicht kommt, sagt der Rabbi zu Elias: Messias hat gelogen; er sagte, er käme heute. Antwortet Elias: er meinte: [...] , heute, wenn ihr auf meine Stimme hört' (Zitat Psalm 95,7). ${ }^{218}$

216 Stéphane Mosès, Von der Zeit zur Ewigkeit. Erlösung - eine problematische Kategorie bei Franz Rosenzweig, in: Gotthardt Fuchs (Hg. u.a.), Zeitgewinn. Messianisches Denken nach Franz Rosenzweig, Frankfurt a.M. 1987, 154. 217 Ebd., 155.

${ }^{218}$ GS I, 344f. - Vgl. auch Hermann Cohen, Die Messiasidee, in: Jüdische Schriften (Hg. B.Strauss), Bd.1, Berlin 1924, 105-124, insbesondere 120. 
Danach erzählt Rosenzweig eine kleine Geschichte über seinen Lehrer Hermann Cohen und die messianische Hoffnung bei den Juden: Er habe Cohen einmal gefragt: „Glauben Sie, Herr Professor, dass das messianische Zeitalter in den nächsten 50 Jahren anbrechen wird?" Darauf antwortete dieser: ,Nein, aber in den nächsten hundert' - eine Geschichte“, fährt Rosenzweig fort, „die doch fast ebenso schön ist wie die ,Heute'-Geschichte“.

Und er unterscheidet anschliessend zwischen „dem Heute, das nur die Brücke zum Morgen sein will, und dem andern Heute, das das Sprungbrett zur Ewigkeit ist [...]“. Es stehe „keinem Tage an der Stirn geschrieben, ob er dieses oder jenes Heute ist. ,Man kann nie wissen'“. ${ }^{219}$ Das ,Man kann nie wissen' drückt nach Rosenzweig die wesentliche, aus der Ungewissheit im jüdischen Messianismus resultierende Ungeduld in der Erwartung eines gänzlich anderen Weltzustandes aus.

Im Unterschied hierzu ist sei die Erfahrung des linearen Fortschreitens der Zeit von Trostlosigkeit, ja Verzweiflung geprägt, weil es zwischen zwei Augenblicken keinen qualitativen Unterschied geben kann, und jedes Heute dem Morgen und Gestern ähnlich ist:

Ohne diese Vorwegnahme und den inneren Zwang dazu, ohne das „Herbeiführenwollen des Messias vor seiner Zeit“ und die Versuchung, das „Himmelreich zu vergewaltigen“, ist die Zukunft keine Zukunft, sondern nur eine in unendliche Länge hingezogene, nach vorwärts projizierte Vergangenheit. Denn ohne solche Vorwegnahme ist der Augenblick nicht ewig, sondern ein sich immerwährend Weiterschleppendes auf der langen Heerstrasse der Zeit (253f.).

Michael Löwy ist der Überzeugung, dass Rosenzweig hier die radikale Kritik am linearen Fortschrittsbegriff vorwegnimmt, die Walter Benjamin in seinen Thesen Über den Begriff der Geschichte (1940) formuliert hat. ${ }^{220}$

${ }^{219}$ GS I, 344f.

${ }^{220}$ Michael Löwy, Erlösung und Utopie, Jüdischer Messianismus und anarchistisches Denken, Berlin 1994, 83. Löwy stellt fest, dass Rosenzweig wie Benjamin die Idee eines unendlichen Fortschritts in der Geschichte durch die jüdische Idee ersetzen wolle, dass jeder Augenblick die Fülle der Ewigkeit in sich aufnehmen könne. Dies stimme fast wörtlich mit der im Anhang der geschichtsphilosophischen Thesen vertretenen Ansicht Benjamins überein. Er führt folgende Stellen bei Walter Benjamin an: Ders., Über den Begriff der Geschichte, in: Sprache und Geschichte, Philosophische Essays, Stuttgart 1992, 141-154, hier 153, These A: „dass für die Juden in der Gegenwart der „Jetztzeit' [...] Splitter der messianischen“ Zeit „eingesprengt“ sind, und dass die Zukunft gemäss jüdischer Auffassung keine homogene und leere Zeit meint, sondern „jede Sekunde“ als „ kleine Pforte“ betrachtet, „durch die der Messias treten könnte“. (These B, ebd., 154.) 


\section{Auf der Schwelle zum zeitlosen Werden Gottes in der Ewigkeit der Überwelt}

Im Übergang zum dritten und letzten Teil von Rosenzweigs Hauptwerk, in einem kurzen Kapitel mit dem Titel Schwelle, wird die im letzten Buch des zweiten Teils bestimmte Ewigkeit der Zukunft in diejenige Ewigkeit überführt, die vollkommen ausserhalb der Zeit ist.

Bereits im Buch über die Erlösung heisst es, dass das ,Wachstum' des Gottesreichs kein ,Zeitmass' habe. Aber der Schwerpunkt von Rosenzweigs Ausführungen liegt, wie wir gesehen haben, auf einem Begriff von Geschichte, der im Zeichen der Zukunft steht. Es geht um die Bestimmung des vom Anspruch der Nächstenliebe geleiteten Handelns und Wirkens in der Welt, die noch kommen soll. Dennoch spielt bereits hier der Begriff der Ewigkeit Gottes, auf dessen Explikation der dritte Teil des Stern der Erlösung abhebt, eine entscheidende Rolle:

Von Gott also nimmt die Erlösung ihren Ursprung, und der Mensch weiss weder Tag noch Stunde. Er weiss nur, dass er lieben soll und stets das Nächste und den Nächsten; und die Welt, sie wächst in sich nach scheinbar eigenem Gesetz; und ob sich Welt und Mensch nun heute finden oder morgen oder wann - die Zeiten sind unberechenbar, sie weiss nicht Mensch noch Welt; die Stunde weiss allein ER, der das Heute jeden Augenblick erlöst zur Ewigkeit. Die Erlösung ist also Ende, vor dem alles Angefangene in seinen Anfang zurücksinkt. Nur dadurch ist sie Voll-endung. (269)

Auf der Schwelle zum dritten Teil des Stern der Erlösung geht es Rosenzweig nun vorwiegend darum, die Ewigkeit Gottes, die auf den Augenblick bezogen und dabei stets im Werden begriffen ist, vom ,Immerwährenden' der als ,zeitlos' attackierten Vorwelt des ersten Teils seines Hauptwerkes abzugrenzen und zugleich in Beziehung zu setzen. Das ,Immerwährende' wird quasi im Sinne Hegels , aufgehoben', d.h. zugleich ,bewahrt' und unter neuen Gesichtspunkten auf einer qualitativ anderen Erkenntnisstufe betrachtet. Gott sei

von Anfang an und [...] in jedem Augenblick und [...] immer im Kommen; und nur wegen dieses Zugleichs seines Immerwährend-, Allzeitund Ewigseins muss man das Ganze als ein Werden bezeichnen. (287f.)

Die auf die Bahn des Beziehungsreichtums in Schöpfung, Offenbarung und Erlösung geratenen Elemente traten, so lautet nun Rosenzweigs Zusammenfassung, „in die Form der Zeitlichkeit“ ein, die für sie selber „den Weg zur Ewigkeit bedeutet“ (288). Dieser Weg führt sie von der Einheit der traditionellen All-Philosophie zu einer neuen Einheit, zur Einheit Gottes, die den Punkt bezeichnet, „der schon so jenseits der ,Bahn' liegt wie ihr göttlicher Ursprung 
jenseits ihres Anfangs“. Die all-umfassende Einheit der Ewigkeit Gottes im neuen Denken, die mit der Tradition herkömmlicher Metaphysik und Ontologie bricht, ist Einheit nicht, indem sie „ist“, sondern „nur indem sie wird“ (287).

Rosenzweig erläutert hier den letzten Schritt seiner existentiell-religionsphilosophischen Umdeutung der ,Zeitlosigkeit' des griechisch-idealistischen All-Denkens in die wahre Ewigkeit Gottes. Er grenzt sich von Hegels Dialektik ab, die das ,Sein' als ,Kugel' auffasst. Und er prangert die ,schlechte', da „in sich selber zurückgekrümmte Unendlichkeit des Idealismus“ an (283f.), um ihr die Wahrheit des im Stern der Erlösung entwickelten Ewigkeitsbegriffs entgegen zu halten. Dieser führt uns in die Überwelt, deren Ewigkeit - wie das Immerwährende der dunklen Vorwelt - von „Schweigen“ erfüllt ist. Aber nun handelt es sich nicht um die Lautlosigkeit der mathematischen Sprache, um das stumme Selbst oder um die Einsamkeit des Helden in der griechischen Götterwelt. Das Schweigen der Überwelt ist vielmehr erleuchtet, weil sie über das Wunder des geschichtlichen Ereignisses der Offenbarung hinausweist in die Vollendung einer erlösten Welt. Wir stehen nach Rosenzweig an der „Schwelle vom Wunder zur Erleuchtung“, die nur in der ,Schau’ erfassbar ist (291).

\section{Weiterführende Lektüre}

Bertolino, L., (2020), Bach in die Synagogen!“. Erlösende Noten in Franz Rosenzweig, in: Naharaim 14 (2020) 209-223.

Dubbels, E., (2011), Figuren des Messianischen in Schriften deutsch-jüdischer Intellektueller 1900-1933, Berlin, De Gruyter.

Scharf, O., (2019), Thinking in Translation: Scripure and Redemption in the Thought of Franz Rosenzweig, Berlin, De Gruyter. 